\title{
TESTE DE CANCELAMENTO EM PACIENTES COM AVC OU TCE: UMA REVISÃO DA LITERATURA
}

\author{
Luara de Freitas Calvette ${ }^{1}$ \\ Camila Rosa de Oliveira ${ }^{2}$ \\ Karina Carlesso Pagliarin ${ }^{3}$ \\ Rochele Paz Fonseca ${ }^{4}$
}

Resumo: O objetivo deste estudo foi identificar e caracterizar testes de cancelamento que são administrados em pacientes que sofreram traumatismo cranioencefálico (TCE) e acidente vascular cerebral (AVC). Revisaram-se todos os tipos de intervenções em que testes de cancelamento eram descritos na população estudada. Foram realizadas buscas com palavras-chave com paradigmas de cancelamento, AVC e TCE nas bases de dados: PubMed, PsycINFO e LILACS. De 216 resumos inicialmente encontrados, após análise de critérios de exclusão, 86 estudos completos foram analisados. Foram encontrados 26 paradigmas de cancelamento, sendo que o Star Cancellation foi mais amplamente utilizado seguido do Line Bissection, Line Crossing e Letter Cancellation. Em indivíduos com AVC o instrumento mais usado foi o Line crossing, e em TCE o Teddy Bear Cancellation Task. Desta forma, os achados sugerem que testes de cancelamento em pacientes com AVC são utilizados principalmente para diagnosticar heminegligência enquanto que em pacientes com TCE são avaliados com paradigmas com perfil atencional.

Palavras-chave: Acidente vascular cerebral; Traumatismos encefálicos; Avaliação; Atenção.

Cancellation test in patients with stroke or TBI: a review of the literature (Abstract): The aim of this review is to identify and characterize cancellation tests

${ }^{1}$ Psicóloga. Mestre em Psicologia (Cognição Humana). Correio electrónico: luaracalvette@hotmail.com

2 Psicóloga. Mestre em Psicologia (Cognição Humana).

${ }^{3}$ Fonoaudióloga. Doutoranda bolsista CNPq do Programa de Pós-Graduação em Psicologia da PUCRS.

${ }^{4}$ Psicóloga e Fonoaudióloga. Prof. Adjunta da Faculdade de Psicologia e do Programa de Pós-Graduação em Psicologia, Área de Concentração Cognição Humana da PUCRS, Bolsista Produtividade $2 \mathrm{CNPq}$ 
that are administered to patients who have suffered a traumatic brain injury (TBI) or a stroke. We reviewed all types of interventions in which cancellation tests were described in the study population. Studies were searched on the databases PubMed, PsycINFO and LILACS, by means of keywords representing three main constructs: cancellation paradigms AND [stroke OR TBI]. Among 216 abstracts initially found, 86 full text articles were included. We found 26 cancellation paradigms. Star Cancellation was the most widely used, followed by Line Bissection, Line Crossing and Letter Cancellation. In individuals with stroke the most used instrument was Line Crossing, and in TBI patients, the Teddy Bear Cancellation Task. Then, general findings suggested that cancellation tests in stroke patients are used mainly to diagnose hemineglect, while TBI patients have been assessed by these paradigms towards an attention profile.

Keywords: Stroke; Traumatic brain injury; Evaluation; Attention.

\section{Introdução}

O acidente vascular cerebral (AVC) e o traumatismo cranioencefálico (TCE) estão entre as doenças neurológicas que mais causam incapacidade física e disfunção cognitiva em diversos países, dentre eles o Brasil (Feijó \& Portela, 2001; Hoffmann, Schmitt \& Bromley, 2009; Piras et al., 2004). Dentre as funções cognitivas comumente alteradas no AVC destacam-se a memória, atenção, linguagem e orientação, enquanto que pacientes com TCE têm usualmente déficits em funções executivas, assim como atenção, memória e velocidade de processamento (Larson, Demery, Cole \& Perlstein, 2005; Draper \& Ponsford, 2008; Mathias \& Wheaton, 2007; Tatemichi et al., 1994). Associado a essas disfunções, os pacientes que sofreram AVC e TCE em geral apresentam sequelas neurocomportamentais tais como dificuldade em se manter empregado e preservar relações sociais (Fann, Hart \& Schomer, 2009), além de transtornos psiquiátricos, estando entre os mais comuns depressão e ansiedade.

Mais especificamente, como resultado de um AVC, devido à complexidade das redes neurais envolvidas nos diferentes processamentos cognitivos, eventos hemorrágicos ou isquêmicos que ocorrem em uma distribuição vascular específica podem afetar mais de uma função cognitiva, por envolver complexas conexões corticais e subcorticais (Ferro, 2001; Rovira, Grive, Rovira \& Alvarez-Sabin, 2005). De acordo com Tatemichi et al. (1994) e Donovan et al. (2008), os infartos de hemisfério esquerdo da artéria cerebral média (ACM) podem resultar principalmente nos seguintes déficits: afasia, mutismo, apraxia bucofacial, agrafia, acalculia, apraxia, e desorientação 
direita/esquerda. Infartos de hemisfério direito da ACM podem resultar, por sua vez, em heminegligência $(\mathrm{HN})$, déficit visuoespacial e visuoconstrutivo, aprosódia, alterações comunicativas, prosopagnosia e anosognosia. Já as lesões pós TCE, resultam em danos difusos, predominantemente afetando as regiões frontal e temporal, produzindo um modelo distinto de déficits cognitivos e comportamentais, que varia de acordo com a localização e a severidade da lesão (National Institute of Health, 1998). Na maioria dos pacientes (90\%) ocorre TCE fechado em que o crânio não é penetrado, e tais danos normalmente são focais, sobrepostos em difusão da substância branca e dano no tronco cerebral (Henry \& Crawford, 2004). Dentre os déficits já apresentados, os danos associados aos aspectos atencionais e perceptivos serão o enfoque nesse trabalho.

A atenção seletiva ou atenção concentrada pode ser definida como habilidade de processar informação selecionada ou relevante sem interferência de informação irrelevante, déficits marcantes nos quadros de AVC e TCE (Schmitter-Edgecombe \& Kibby, 1998). Para avaliar esta função neuropsicológica são utilizados testes de cancelamento visual, os quais envolvem busca e escaneamento visual de letras ou símbolos, concomitantemente ou não a demais distratores, que estão organizados em colunas ou linhas ou de forma pseudoaleatória. Tarefas de cancelamento que usam disposição pseudoaleatória de símbolos complexos são mais difíceis de serem realizados do que os testes nos quais os estímulos-alvo estão organizados em linhas ou colunas (Lowery, Ragland, Gur, Gur \& Moberg, 2004). Alguns dos instrumentos amplamente utilizados na avaliação de ambos os quadros é o teste de cancelamento de linhas (Leibovitch et al.,1998; Maeshima et al., 1997; Mark \& Heilman, 1998; Vanier et al.,1990) e Letter Cancellation (Hallingan \& Marshall, 1998; Marshall, Grinnell, Heisel, Newall \& Hunt, 1997). Como o desempenho em tarefas que avaliem atenção seletiva é fundamental para o funcionamento do indivíduo em uma variedade de situações do dia a dia, uma compreensão da relação desta com o AVC e TCE torna-se necessária.

Outro aspecto importante está relacionado com indivíduos que sofreram em sua maioria e com mais frequência AVC ou TCE de hemisfério direito, em que ocorre um quadro complexo denominado $\mathrm{HN}$ unilateral ou hemi-atenção (Beis et al., 2004; Gianotti, Messerli \& Tissot, 1972). Nesses casos, o paciente ignora, ou não responde a estímulos contralaterais ao lado em que ocorreu a lesão cerebral (Azouvi et al., 2006; Vossel et al., 2011). Esta predominância é baseada na concepção de que o hemisfério direito (HD) distribui a atenção uniformemente e dirige o foco para ambos os lados do espaço extrapessoal, enquanto o hemisfério esquerdo (HE), dirige predominantemente para o lado contralateral (Mesulam, 1999). Portanto, os testes de cancelamento também podem ser utilizados para avaliar heminegligência visual. Esses pacientes tipicamente mostram dificuldades em orientação 
espacial ou atenção seletiva em direção ao hemiespaço contralesional (Siéroff, Decaix, Chokron \& Bartolomeo, 2007). Em estudos que investigam as variáveis que influenciam na evolução do tratamento pós-lesão neurológica, a heminegligência, quando presente, ocasiona um pior prognóstico (Fordell, Bodin, Bucht \& Malm, 2011), sendo essencial sua identificação para estimativas de intervenção.

Uma vez que os testes de cancelamento são utilizados para avaliar aspectos atencionais e perceptivos, sendo especialmente úteis para a acurácia diagnóstica da síndrome de heminegligência, entende-se que é necessário realizar um levantamento dos testes de cancelamento que vem sendo mais frequentemente escolhidos nas pesquisas com pacientes com lesão cerebral adquirida. Assim, os achados do presente estudo poderão auxiliar clínicos a selecionarem de modo crítico suas tarefas avaliativas para estes fins. Mais especificamente, uma vez que alterações atencionais e perceptivas têm sido amplamente relatadas nos quadros de AVC e TCE e essas lesões são as que ocorrem com mais frequência na população em geral, acredita-se que é necessário caracterizar esses instrumentos de forma que o clínico possa fazer escolhas baseadas em evidências.

Desta forma, esta revisão teve por objetivo caracterizar quantos e quais os paradigmas de cancelamento visual têm sido utilizados em estudos neuropsicológicos com amostras de pacientes com AVC e TCE. A partir dos resultados espera-se compreender para que fim tais testes de cancelamento têm sido utilizados em ambas as populações neurológicas, os aspectos que envolvem essa avaliação; bem como um entendimento quanto as características atencionais e perceptivas dessas lesões adquiridas.

\section{Método}

A revisão sistemática da literatura foi realizada no mês de janeiro de 2011. Foram encontrados 216 resumos (Figura 1) nas fontes PUBMED, LILACS e PSYCinfo. Essas bases foram utilizadas por abrangerem pesquisas nacionais (Brasil) e internacionais. Nas bases de dados foram realizadas duas buscas, uma com as palavras-chave referentes aos construtos "teste de cancelamento" e "AVC" e outra com os construtos "teste de cancelamento" e "TCE". Os construtos utilizados abrangem os aspectos principais da avaliação por testes de cancelamento em duas populações neurológicas. As palavras foram escolhidas por serem frequentemente utilizadas em artigos sobre a temática e/ou serem descritores nos sites PubMed e LILACS. Não se delimitou a data de publicação dos artigos com o intuito de englobar as pesquisas precursoras na área. A sintaxe utilizada encontra-se na Tabela 1. 
Os critérios de seleção final desses resumos para sua inclusão na revisão sistemática foram os seguintes: 1) abordar estudos com indivíduos com TCE ou AVC; 2) utilizar instrumentos ou tarefas que avaliassem atenção por testes de cancelamento visual. A partir desses critérios, foram excluídos três artigos que não descreviam o instrumento utilizado e um que não era identificada a população estudada. Além disso, os artigos completos dos resumos selecionados após exclusão dos repetidos e dos não localizados (por serem artigos muito antigos e não estarem disponíveis pelo Portal CAPES) foram analisados com ênfase nas seções Objetivo, Método e Resultados e as informações destes tabelados.

Figura 1. Fluxo de análise de resumos e artigos completos nas bases de dados PubMed LILACS e PsycINFO.

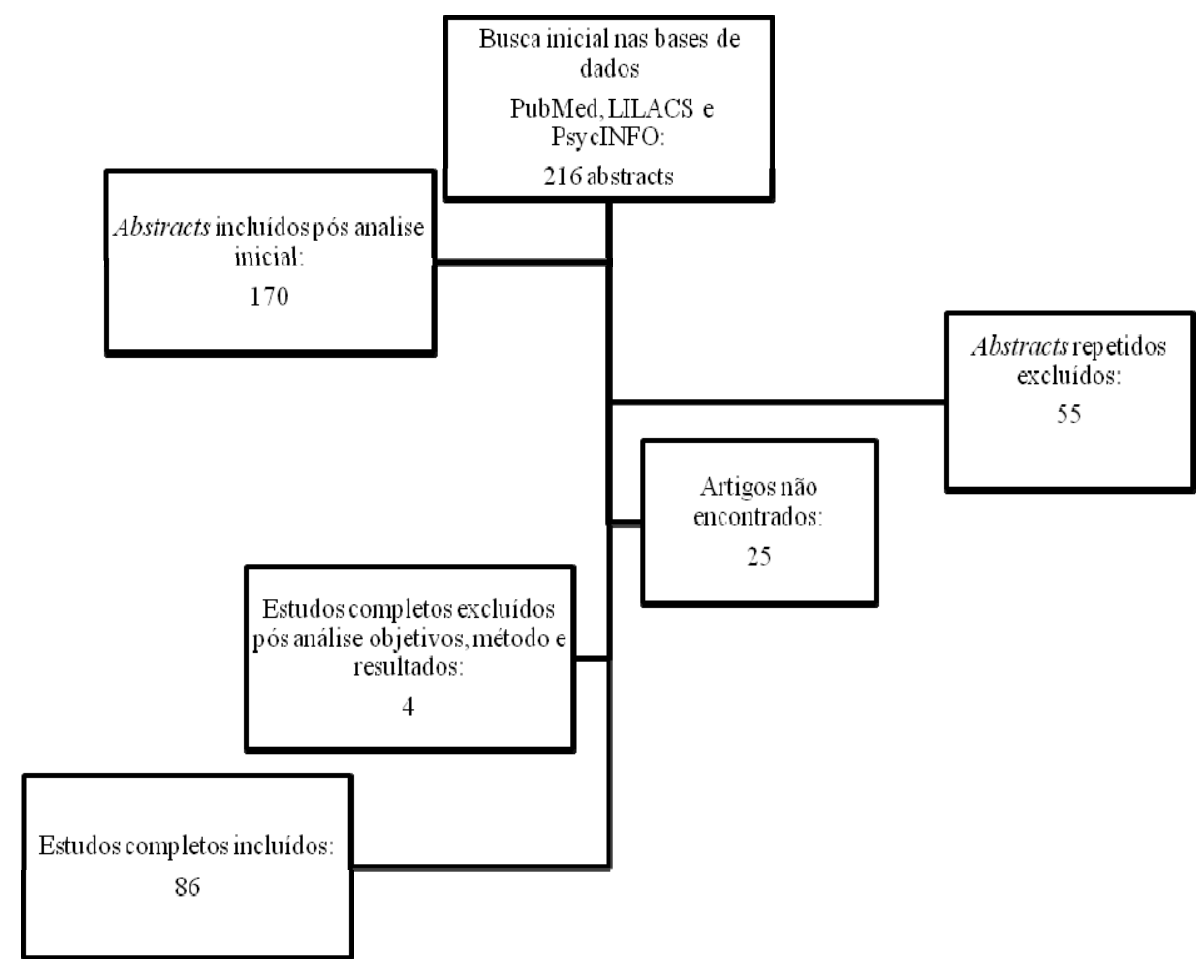




\section{Resultados}

A seguir serão apresentados os resultados por meio de quatro tabelas. A Tabela 1 apresenta a sintaxe utilizada nas buscas realizadas nas bases de dados, bem como a quantidade de resumos identificados na primeira busca e de resumos selecionados após a primeira análise de critérios de exclusão. Em um primeiro momento, tais dados são apresentados para a amostra de AVC. $\mathrm{Na}$ segunda parte da Tabela 1, estudos com amostras com TCE foram quantificados. Ambas foram avaliadas com paradigmas de cancelamento.

Tabela 1. Quantidade inicial e final de resumos analisados de estudos com paradigmas de cancelamento em pacientes com AVC e/ou TCE

\begin{tabular}{|c|c|c|c|}
\hline $\begin{array}{c}\text { Base de } \\
\text { dados }\end{array}$ & Sintaxe para AVC & $\begin{array}{l}\text { Número de } \\
\text { resumos } \\
\text { identificados }\end{array}$ & $\begin{array}{l}\text { Número de } \\
\text { resumos } \\
\text { selecionados }\end{array}$ \\
\hline Pubmed & $\begin{array}{l}\text { "cancellation"[Title/Resumo] OR "can- } \\
\text { cellation task"[Title/Resumo] OR "can- } \\
\text { cellation tasks"[Title/Resumo] OR "can- } \\
\text { cellation test"[Title/Resumo] OR "cancel- } \\
\text { lation tests"[Title/Resumo] AND "hemi- } \\
\text { sphere brain damage"[Title/Resumo] OR } \\
\text { "hemisphere brain dam- } \\
\text { aged"[Title/Resumo] OR } \\
\text { "stroke"[Title/Resumo] OR "unilateral } \\
\text { hemispheric damage"[Title/Resumo] OR } \\
\text { "right hemisphere lesion"[Title/Resumo] } \\
\text { OR "right hemisphere } \\
\text { stroke"[Title/Resumo] OR "right hemi- } \\
\text { sphere damage"[Title/Resumo] }\end{array}$ & 114 & 95 \\
\hline \multirow{2}{*}{ Lilacs } & $\begin{array}{l}\text { Português: cancelamento[Pala- } \\
\text { vras] and acidente vascular cere- } \\
\text { bral[Palavras] }\end{array}$ & 0 & 0 \\
\hline & $\begin{array}{l}\text { Inglês: cancellation[Pala- } \\
\text { vras] and stroke[Palavras] }\end{array}$ & 2 & 1 \\
\hline PSYCinfo & $\begin{array}{l}\text { Resumo: "hemisphere brain damage" OR } \\
\text { Resumo: "stroke" OR Title: "unilateral } \\
\text { hemispheric damage" OR Title: "right } \\
\text { hemisphere lesion" OR Title: "right hemi- } \\
\text { sphere stroke" OR Title: "right hemi- } \\
\text { sphere damage" AND Resumo: "cancel- } \\
\text { lation" OR Resumo: "cancellation task" } \\
\text { OR Resumo: "cancellation tasks" OR } \\
\text { Resumo: "cancellation test" OR Resumo: } \\
\text { "cancellation tests" }\end{array}$ & 79 & 61 \\
\hline
\end{tabular}




\begin{tabular}{|c|c|c|c|}
\hline $\begin{array}{c}\text { Base de } \\
\text { dados }\end{array}$ & Sintaxe para TCE & $\begin{array}{l}\text { Número de } \\
\text { resumos } \\
\text { identificados }\end{array}$ & $\begin{array}{l}\text { Número de } \\
\text { resumos } \\
\text { selecionados }\end{array}$ \\
\hline Pubmed & $\begin{array}{l}\text { "cancellation"[Title/Resumo] OR "can- } \\
\text { cellation task"[Title/Resumo] OR "can- } \\
\text { cellation tasks"[Title/Resumo] OR "can- } \\
\text { cellation test"[Title/Resumo] OR "cancel- } \\
\text { lation tests"[Title/Resumo] AND "ac- } \\
\text { quired brain lesions"[Title/Resumo] OR } \\
\text { "traumatic brain injury"[Title/Resumo] } \\
\text { OR "closed head injury"[Title/Resumo] } \\
\text { OR "head injury"[Title/Resumo] }\end{array}$ & 10 & 6 \\
\hline \multirow{2}{*}{ Lilacs } & $\begin{array}{l}\text { Português: cancelamen- } \\
\text { to[Palavras] and traumatismo crania- } \\
\text { no[Palavras] }\end{array}$ & 0 & 0 \\
\hline & $\begin{array}{l}\text { Inglês: cancellan- } \\
\text { tion[Palavras] and traumatic brain in- } \\
\text { jury[Palavras] }\end{array}$ & 0 & 0 \\
\hline PSYCinfo & $\begin{array}{l}\text { Resumo: "acquired brain lesions" OR } \\
\text { Resumo: "traumatic brain injury" OR } \\
\text { Resumo: "closed head injury" OR Re- } \\
\text { sumo: "head injury" AND Resumo: "can- } \\
\text { cellation" OR Resumo: "cancellation } \\
\text { task" OR Resumo: "cancellation tasks" } \\
\text { OR Resumo: "cancellation test" OR Re- } \\
\text { sumo: "cancellation tests" }\end{array}$ & 11 & 7 \\
\hline
\end{tabular}

O número total de artigos completos analisados nessa revisão sistemática foi de 86. Na Tabela 1 observa-se que o número mais expressivo de publicações sobre avaliação com instrumentos de cancelamento em pacientes com AVC foi na base de dados PubMed. Em contrapartida, na segunda parte da tabela, nota-se que o número mais expressivo de publicações sobre exame com tais paradigmas em indivíduos pós-TCE, foi tanto na base de dados PubMed e PsycINFO. É também importante ressaltar a discrepância entre o número de artigos encontrados com pacientes com AVC daqueles com TCE, sendo a frequência de estudos com a primeira amostra muito maior do que com a última.

A análise dos textos completos foi subdividida em duas tabelas, sendo que a Tabela 2 descreve os paradigmas de cancelamento utilizados na avaliação das populações neurológicas enfocadas. Quantificou-se o número de estudos por instrumento utilizado, especificando-se a quantidade de investigações para cada amostra clínica, AVC e/ou TCE. Em complementaridade, na Tabela 3, as investigações são agrupadas por seu objeto de estudo: função ou síndrome neuropsicológica avaliada pelos paradigmas de cancelamento. 


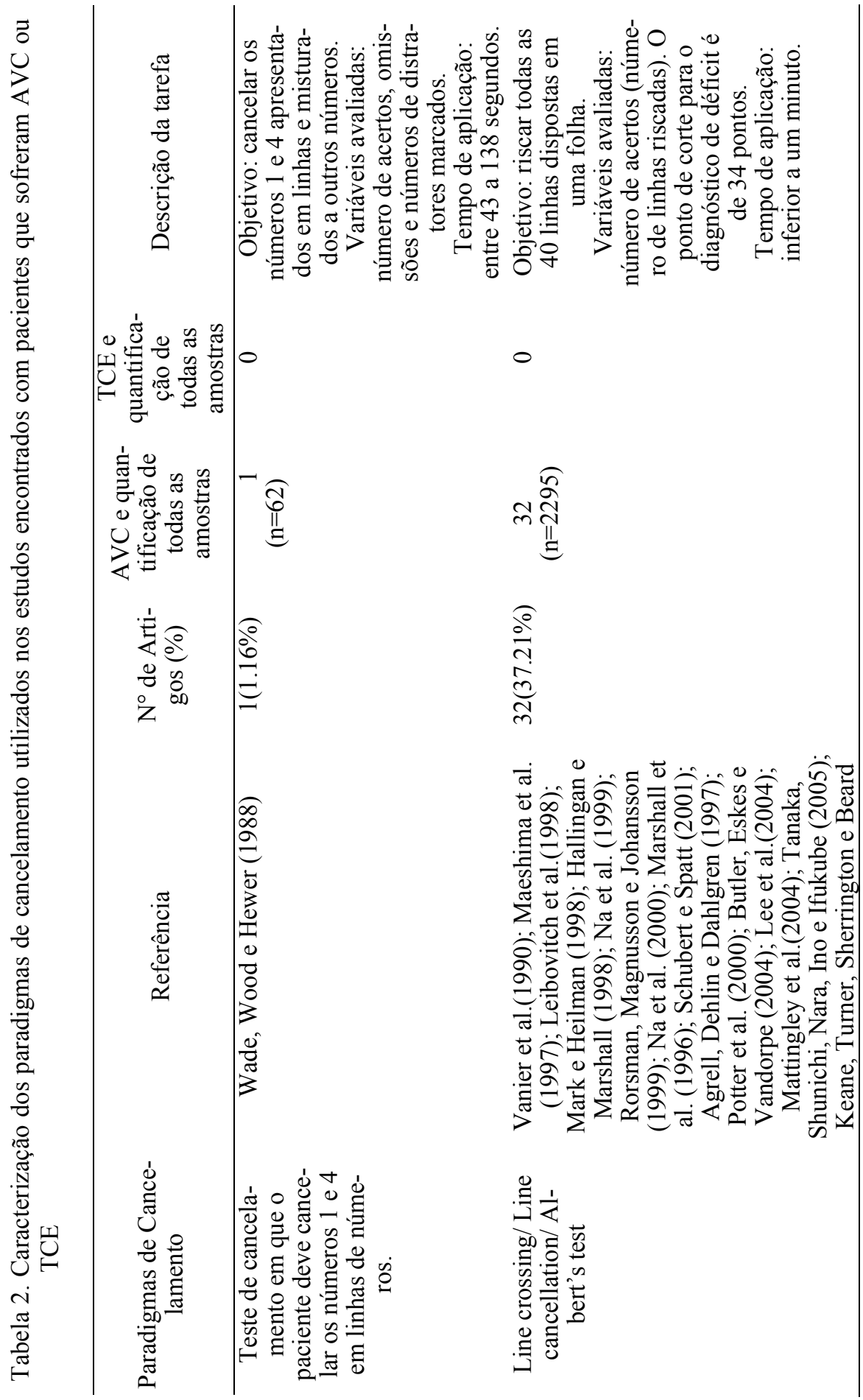




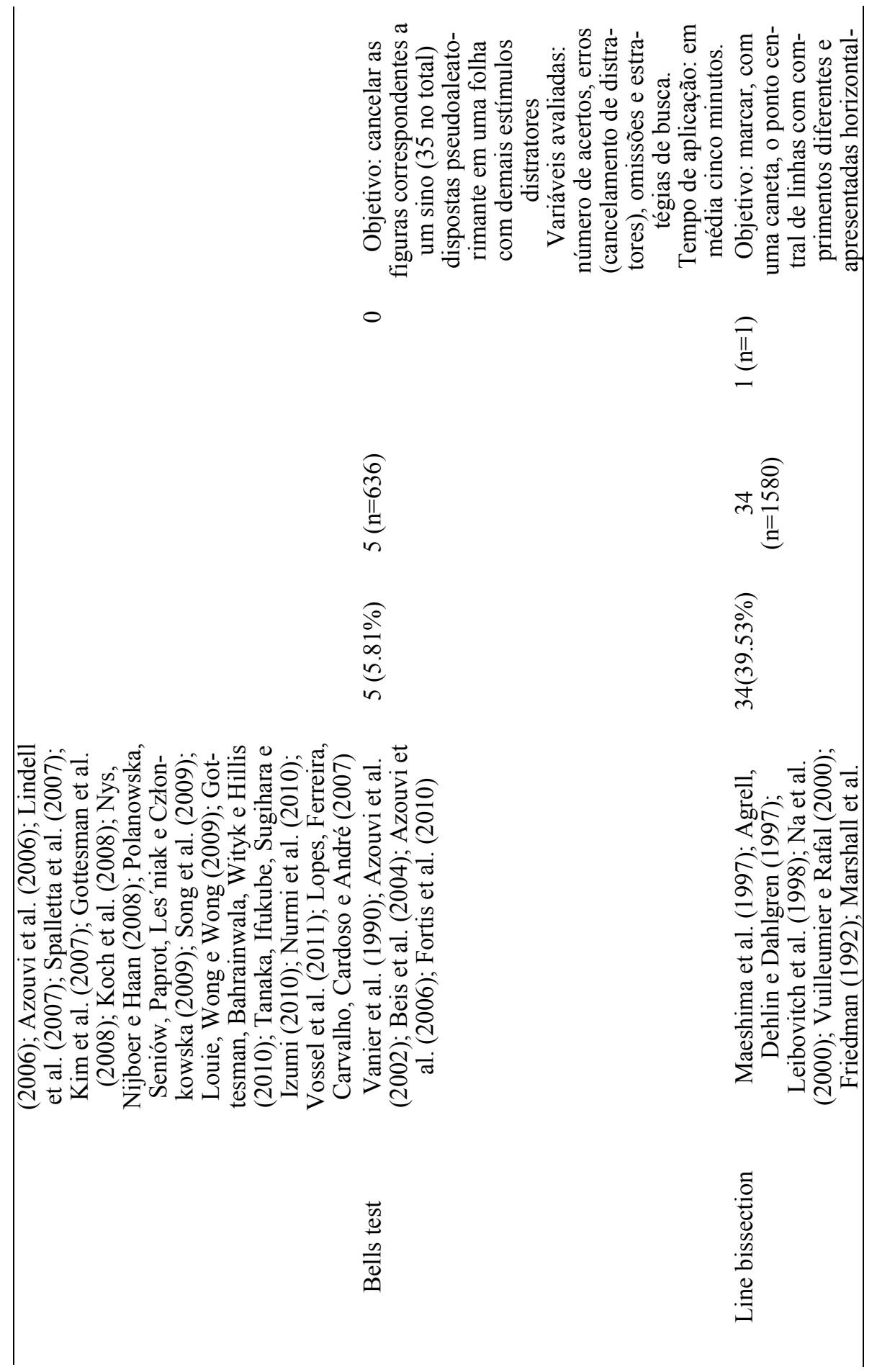



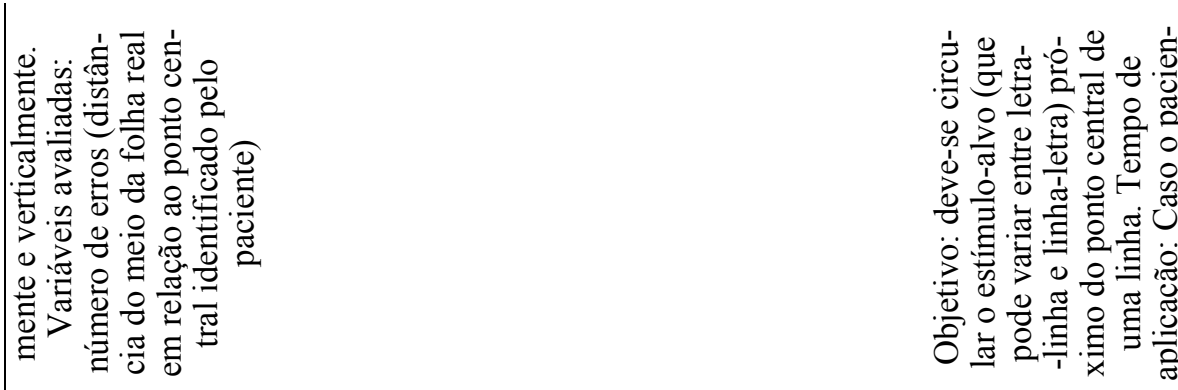

$\circ$

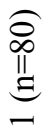

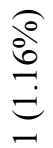

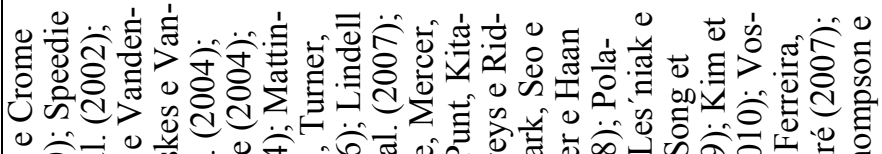

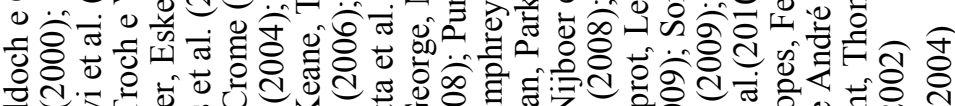

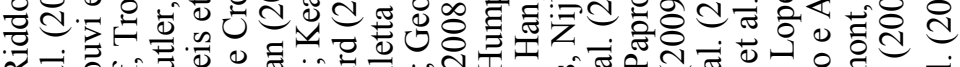
व

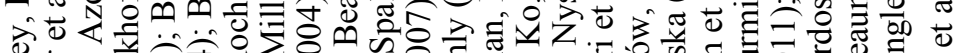

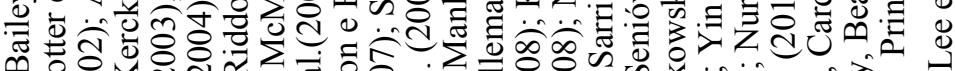

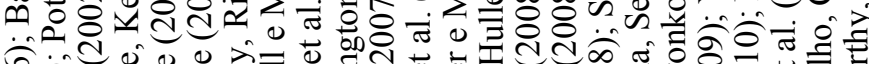

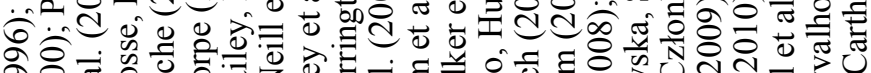

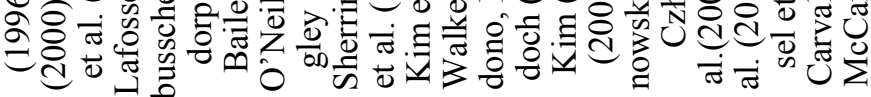

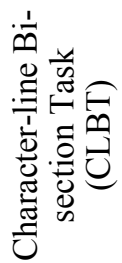




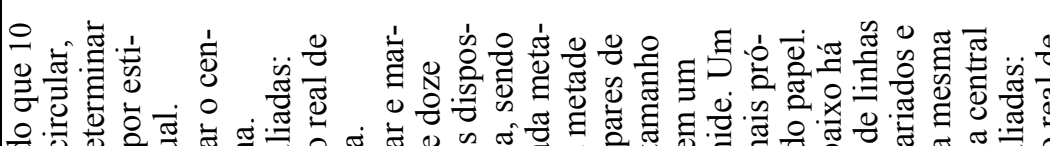

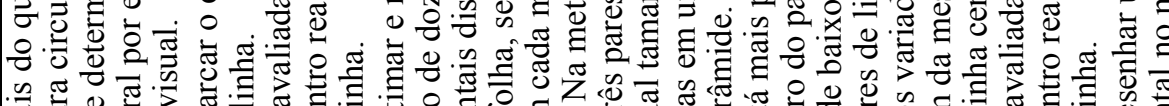

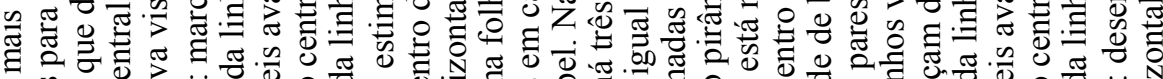

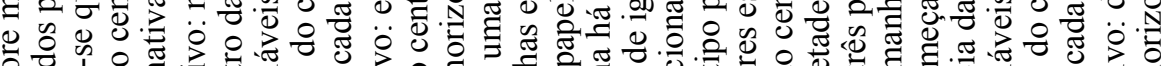

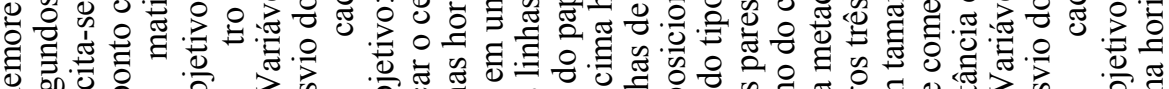

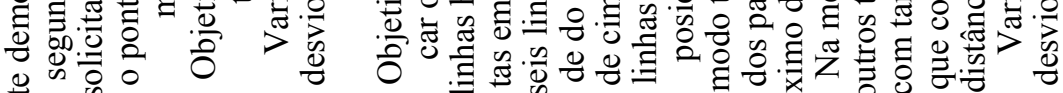

$0 \quad 0$

0

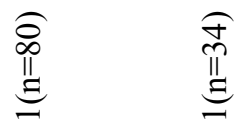

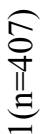

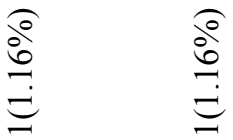

กิ

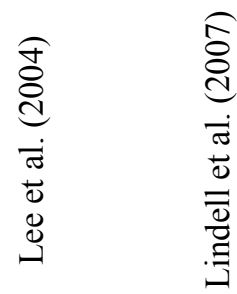

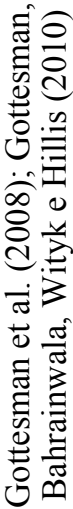

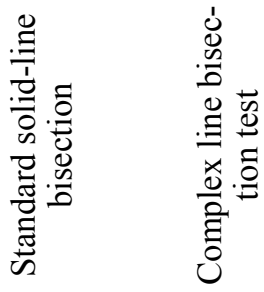

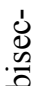

$\stackrel{\mathscr{\Xi}}{.}$.

导 

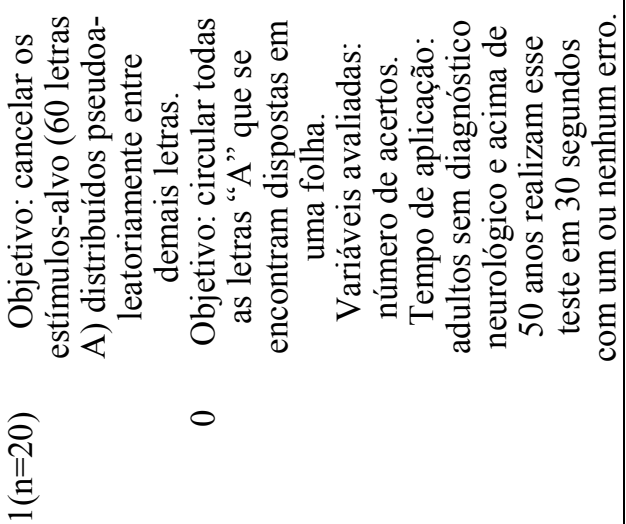

$\frac{2}{\pi}$

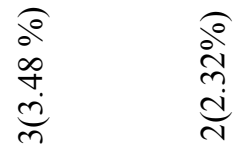

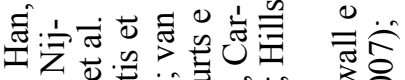

की

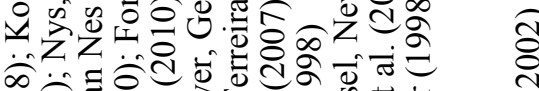

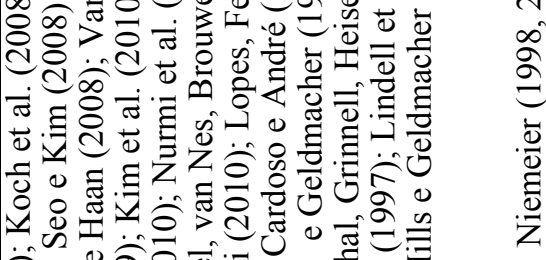

क人

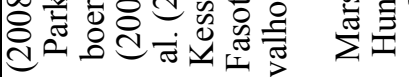

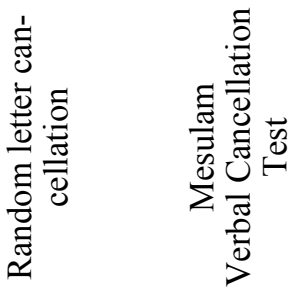




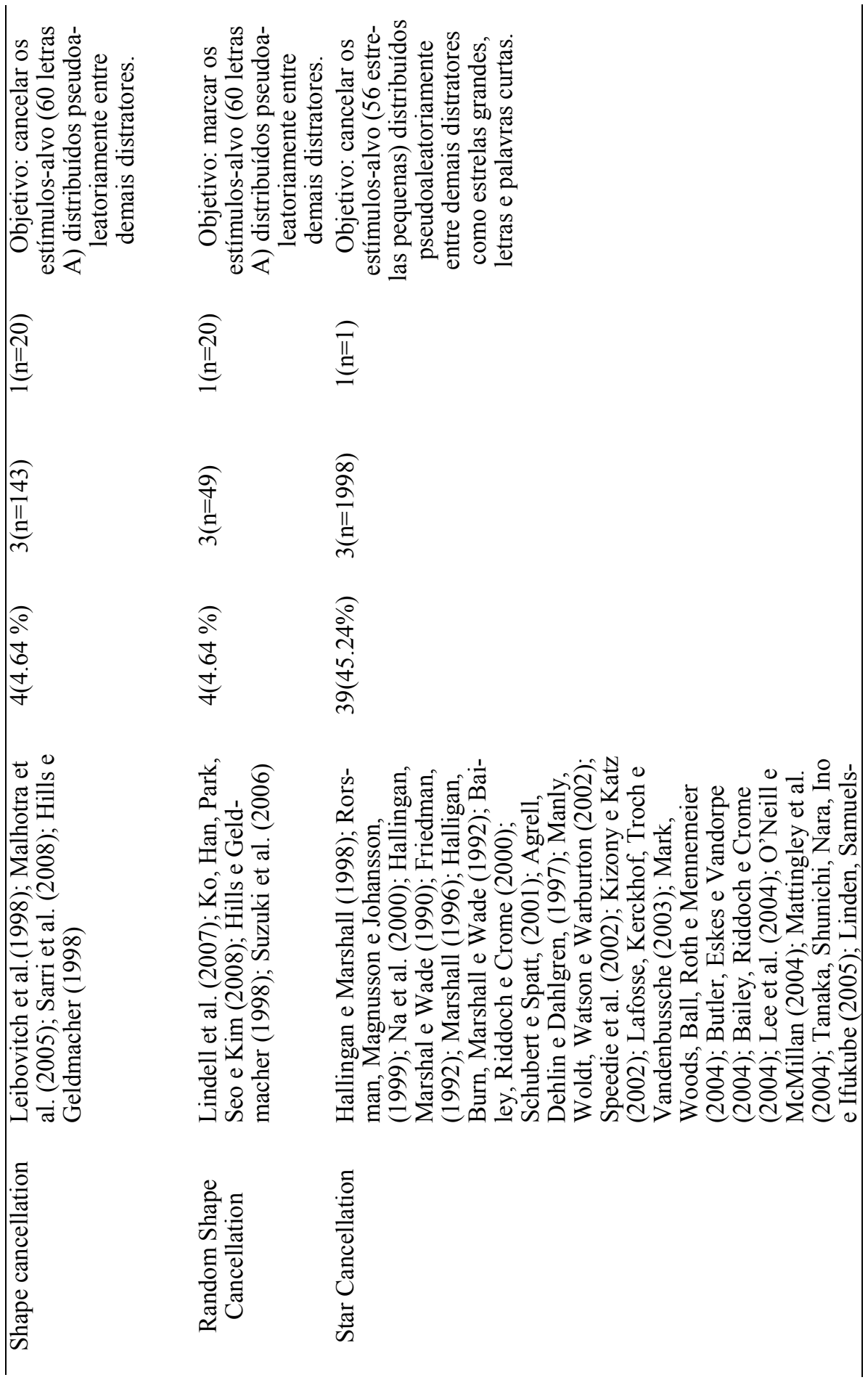




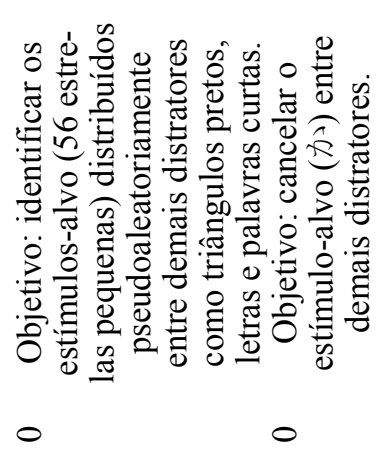

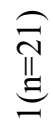

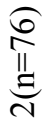

do
¿

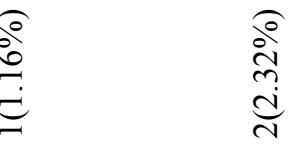

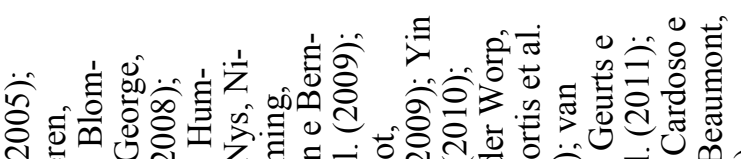

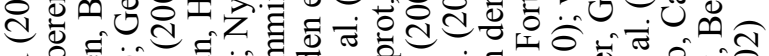
च 픈

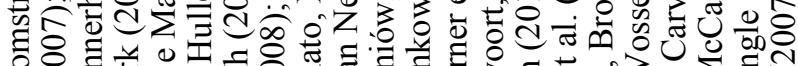

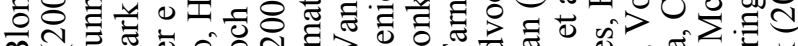

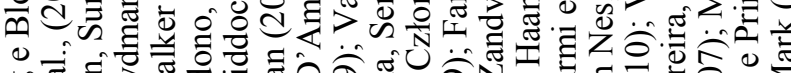

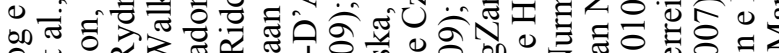

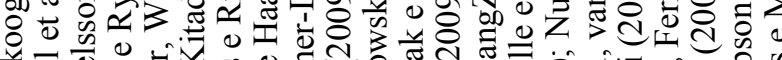
के

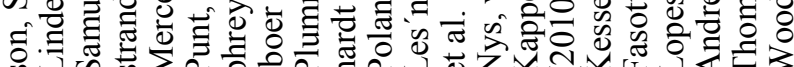

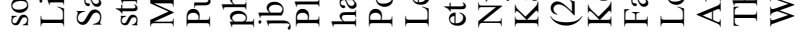
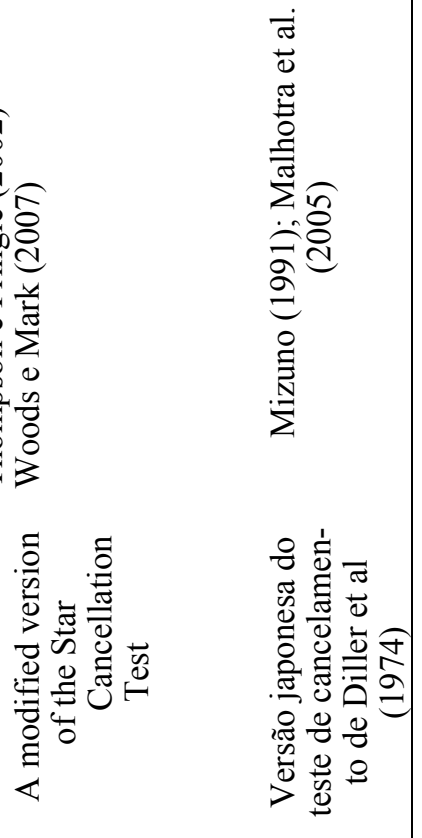


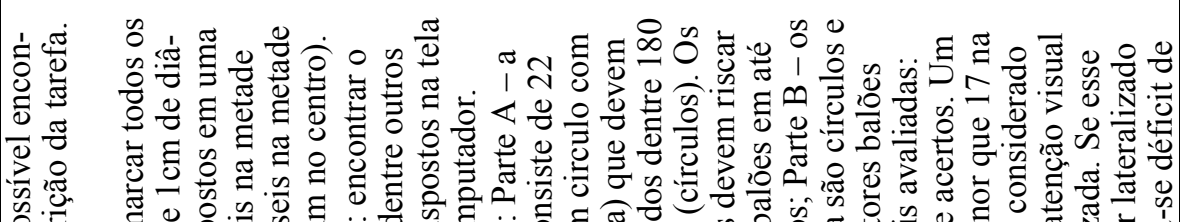

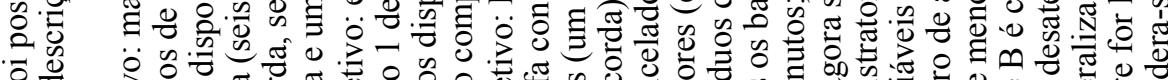
司 它

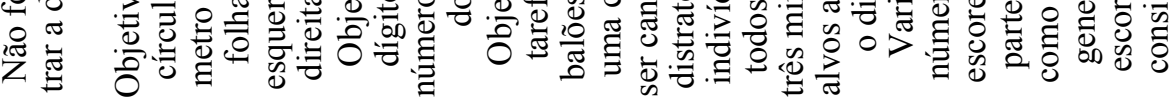
000

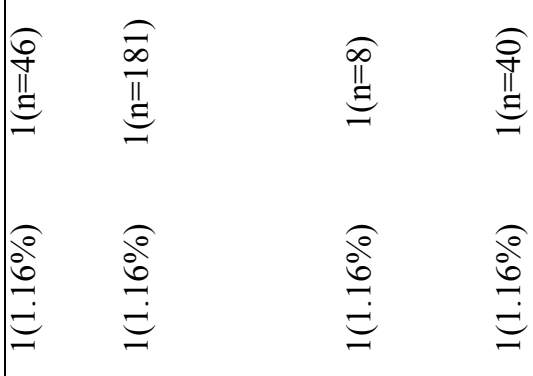

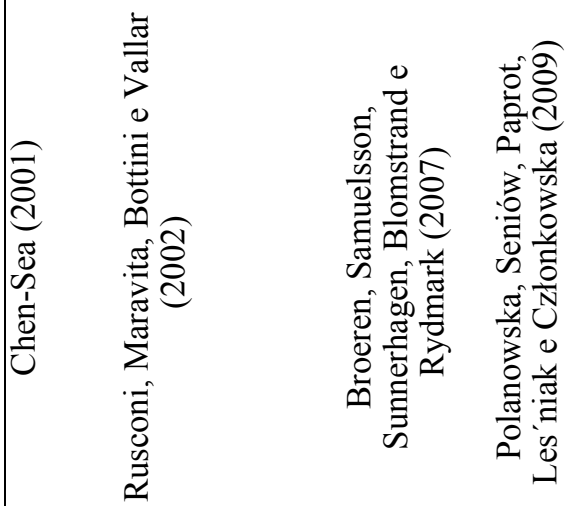

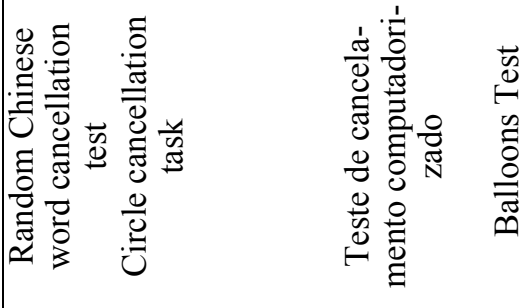




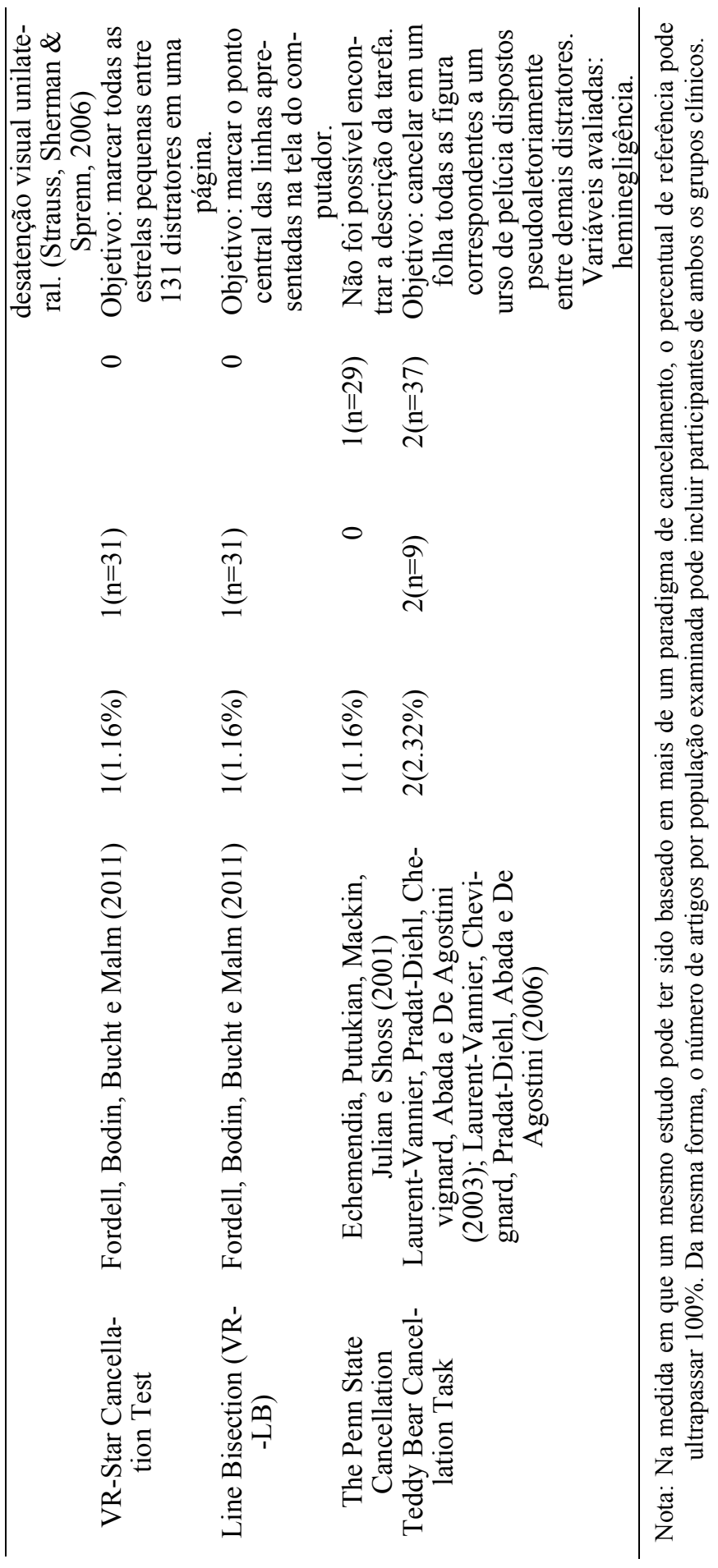


De acordo com a apresentação dos dados na Tabela 2, foram identificados 26 paradigmas de cancelamento. Observa-se que o teste mais amplamente utilizado nas pesquisas é o Star Cancellation seguido do Line Bissection, Line Crossing e Letter Cancellation. O instrumento que foi utilizado para avaliar o maior número de participantes com AVC foi o Line Crossing, pois segundo Ferber e Karnath (2001) é um teste tradicional na avaliação de heminegligência sendo rápido e fácil de administrar já com TCE, foi o Teddy Bear Cancellation Task que consiste em um teste de cancelamento com distratores desenvolvido para crianças baseado no Bells Test para adultos, foi escolhido porque os testes de cancelamento são fáceis de administrar e identificam heminegligência (Laurent-Vannier, Chevignard, Pradat-Diehl, Abada \& De Agostini, 2006).

Ressalta-se, ainda, que grande parte das pesquisas encontradas com AVC $(97.6 \%)$ incluíam participantes adultos e apenas duas com crianças $(\mathrm{n}=6)$. No entanto, das investigações com pacientes com TCE, duas $(40 \%)$ envolviam crianças. Na Tabela 3 os estudos incluídos foram agrupados de acordo com seu foco de interesse de investigação a partir de um ou mais paradigmas de cancelamento visual.

Na Tabela 3 observa-se que o grande enfoque dos autores ao usar testes ou tarefas de cancelamento é identificar heminegligência. Em geral, tal diagnóstico é baseado na análise do número de omissões do campo visual contralateral ao da lesão na folha de resposta. Há também artigos que discutem a velocidade de processamento e o papel da mensuração do tempo na identificação de heminegligência.

$\mathrm{Na}$ Tabela 4 pode-se observar as estratégias utilizadas pelo paciente durante a busca pelos alvos, tais como, coluna ou direção utilizada para o início da busca, distância medida inter alvo e quantificação das intersecções realizadas. Alguns autores também utilizam essas tarefas para avaliar componentes das funções executivas, mais precisamente, organização, planejamento e inibição de estímulos visuais. Portanto, nota-se que apesar do enfoque ser na avaliação da ocorrência de heminegligência, existe uma vasta gama de avaliações qualitativas complementares e mais específicas decorrentes da análise de estratégias e de tipos de erros. 


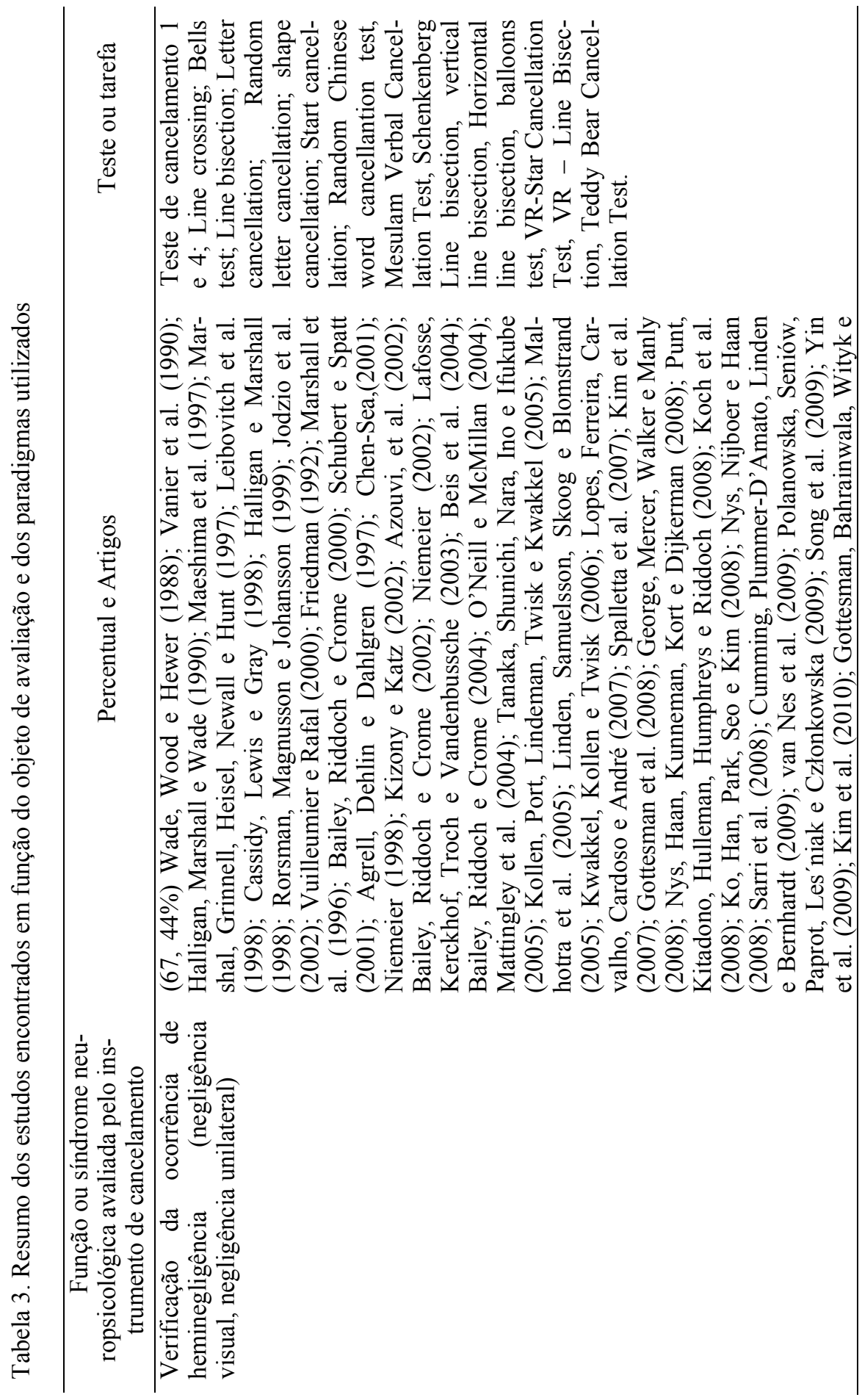




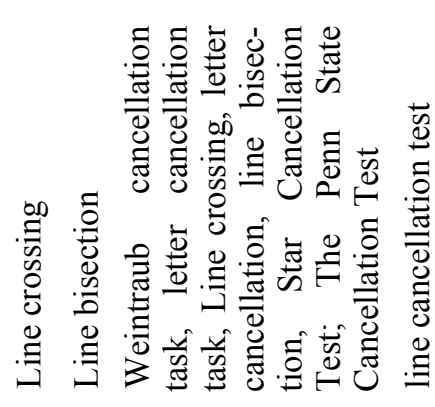

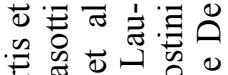

I

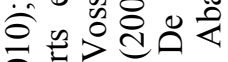

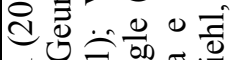

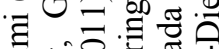

过包完壳

○ ह

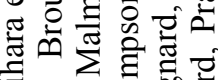

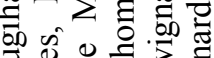

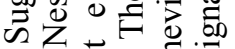

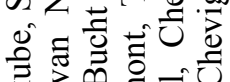

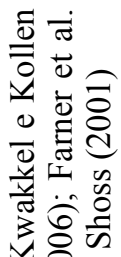

․ํㅇ

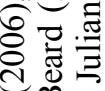

त่

¿ 응

이

츨

क $\frac{\bar{v}}{\sqrt{2}}$

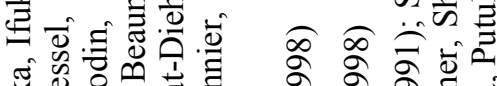

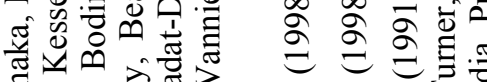

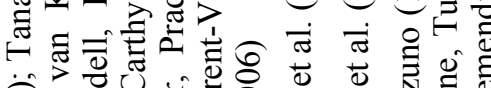

$\ddot{\hat{O}} \cdots \overline{0}$

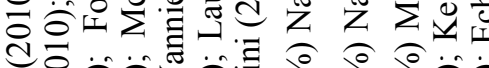

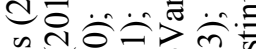

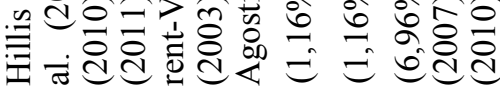

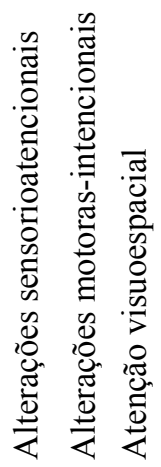




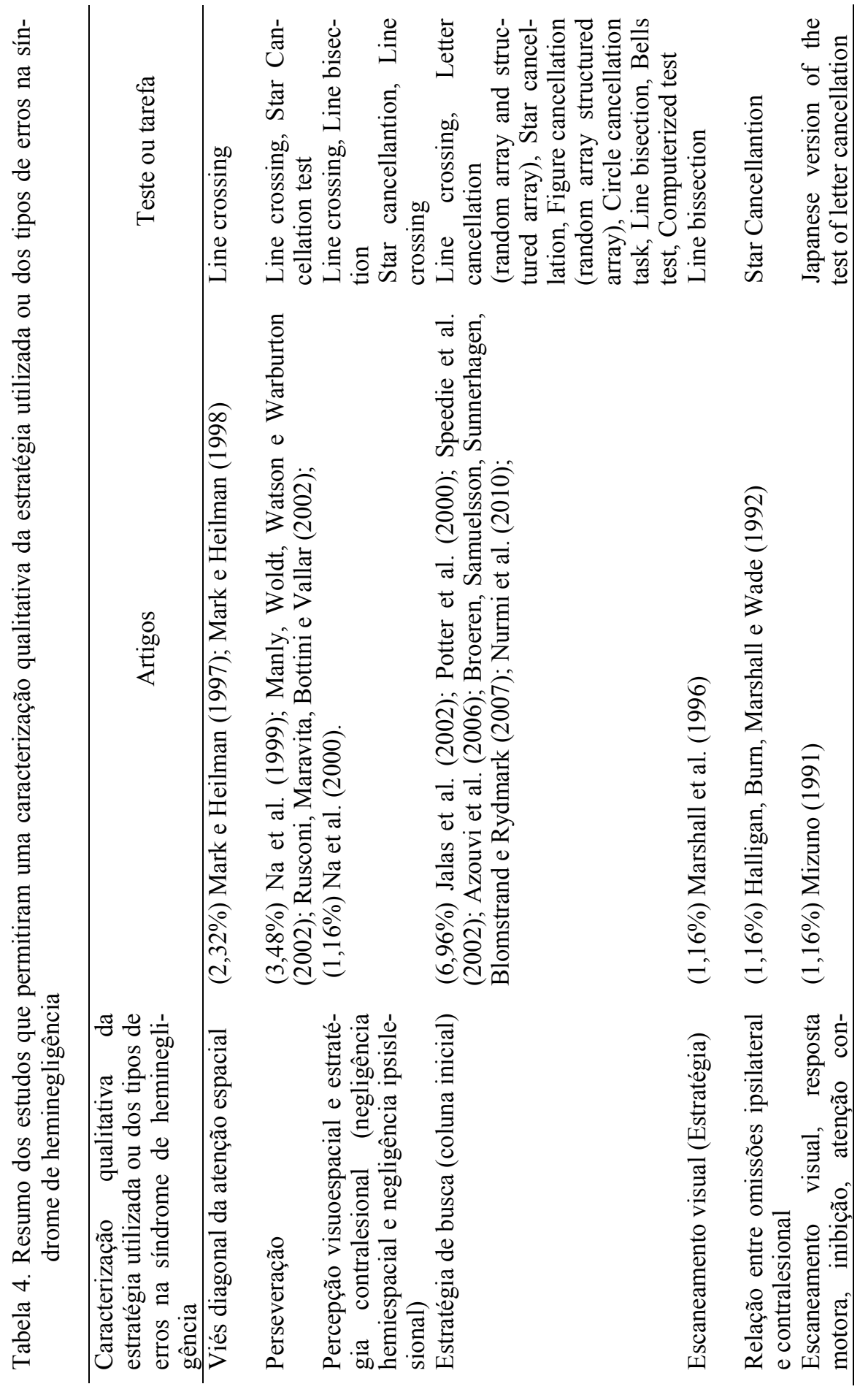




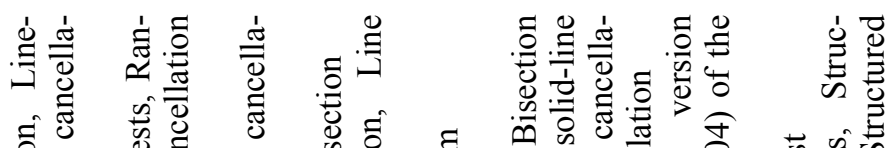

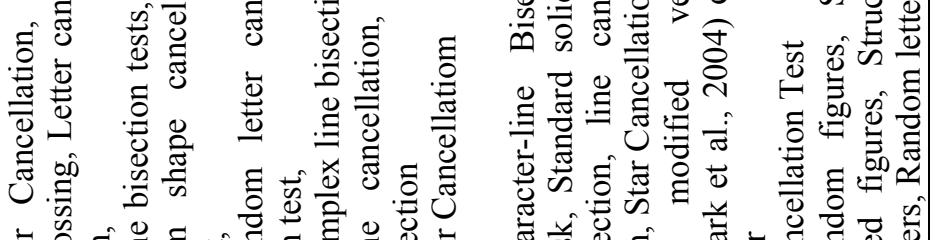
离

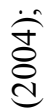

$\dot{\bar{\theta}}$

过

$\stackrel{\Xi}{\supset}$

0

ণ্ঠి

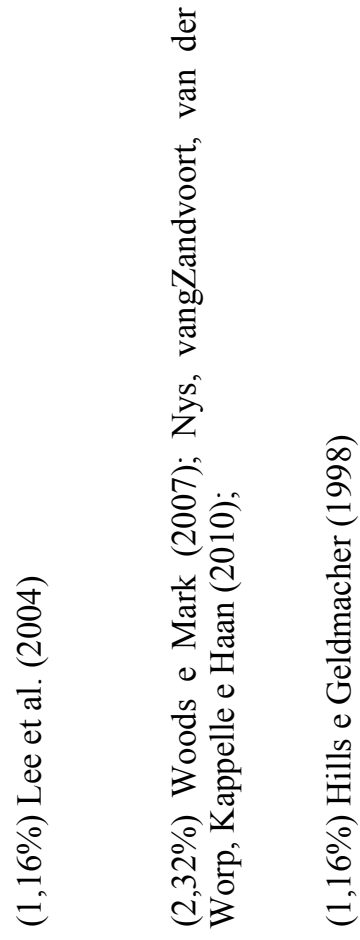

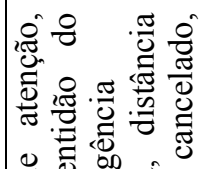

동

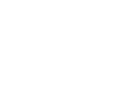

旁

कृ

i $\frac{\dot{\pi}}{4}$

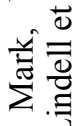

ชิ

กิ

ชิ

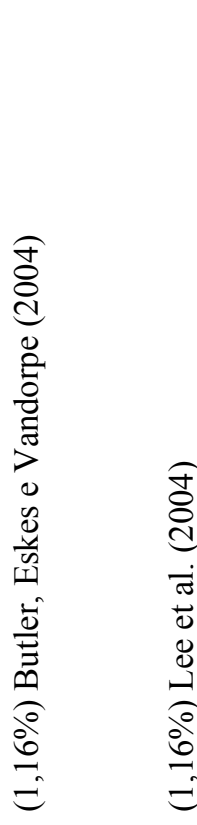

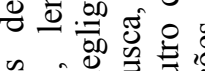

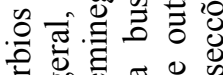

司

음 워 0 올

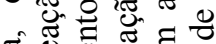

芯

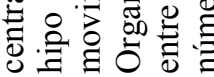

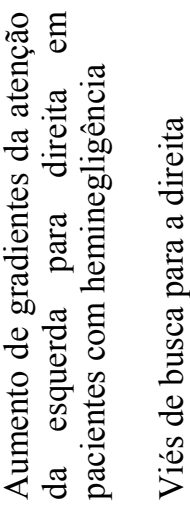

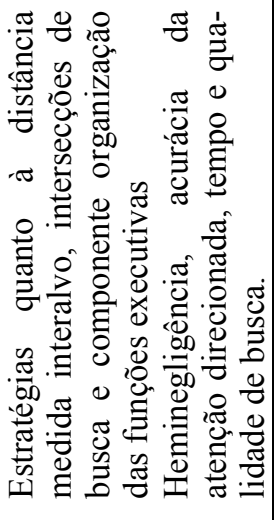




\section{Discussão}

A partir dessa revisão, encontrou-se uma quantidade e variedade importante de paradigmas de cancelamento visual utilizados na avaliação de pacientes com os quadros neurológicos súbitos AVC e/ou TCE. Fez-se um levantamento de 26 instrumentos que vem sendo utilizados para identificar heminegligência, e/ou caracterizar o processamento de atenção visual concentrada associada ou não ao processamento perceptivo. Os instrumentos utilizados tanto pós-TCE quanto pós-AVC foram, Line Bissection, Star Cancellation, Letter Cancellation, Random Letter Cancellation, Shape Cancellation, Random Shape Cancellation e Teddy Bear Cancellation Task. Nota-se, então, que todos são paradigmas específicos de cancelamento de alvos, exceto o Line Bisection. Embora desde o início da década de 1990 não venha mais sendo considerado uma tarefa de cancelamento propriamente dita (Friedman, 1990; Lee et al., 2004), segue sendo incluído em baterias de avaliação da atenção concentrada visual e de heminegligência. O Line Bissection requer que o paciente seccione uma linha em duas de igual tamanho, sendo cada vez mais sensível para o diagnóstico de hemianopsia, em contrapartida, os testes de cancelamento dependem de uma busca visual acurada de alvos dentre vários estímulos, distratores ou não, sem requerer especificamente percepção de tamanho (Ferber \& Karnath, 2001).

O paradigma mais utilizado na avaliação de AVC foi o Star Cancellation que consiste de uma página com 52 grandes estrelas, 10 palavras curtas e 13 letras posicionados de forma aleatória entre 56 pequenas estrelas. $\mathrm{O}$ instrumento está incluído em uma das mais conhecidas baterias para avaliação de heminegligência (Behavioural Inattention Test ou BIT) sendo considerado dentre todos os subtestes incluídos na bateria, o mais sensível (Bailey, Riddoch \& Crome, 2002). Já nos quadros de TCE, o paradigma encontrado com mais frequência foi o Teddy Bear Cancellation Task que é uma tarefa com estímulos distribuídos de forma pseudoaleatória e com distratores, sendo bastante sensível na identificação de heminegligência em criança com lesão cerebral adquirida. Outras informações como escores de omissão e estratégias de busca também podem ser calculados (Laurent-Vannier et al., 2006).

Dos instrumentos elencados na Tabela 2, muitos fazem parte de baterias exclusivas para avaliação de heminegligência o que faz com que alguns testes sejam mais conhecidos e aplicados. Podem-se identificar quatro, sendo elas: Sunnybrook Neglect Battery (SNB) que contêm os subtestes: bateria de desenhos (desenho espontâneo, cópia de um relógio e margarida), Line Cancellation, Line Bisection e Shape Cancellation (Leibovitch et al.,1998); Behavioral Inattention Test (BIT), consiste de seis testes com lápis e papel (Line Crossing, Letter e Star Cancellation, cópia de figura e forma, Line 
Bisection e desenho representacional), e nove testes comportamentais (escaneamento de figura, ligação de telefone, leitura de um menu, falar e marcar o tempo, classificação de moedas, cópia de sentença e endereço, navegação por mapa e classificação de cartas). A bateria pode levar até 45 minutos. Uma bateria breve foi desenvolvida a partir da original sendo mais apropriada para pacientes idosos. Dos testes utilizados nessa bateria, o Star Cancellation Test usado na versão modificada foi o teste mais sensível (Bailey, Riddoch \& Crome, 2000); Batterie d'evaluation de la n'egligence spatiale (BEM): Essa bateria foi desenvolvida para facilitar a avaliação em pessoas com o Francês como idioma. Dessa bateria fazem parte os seguintes testes: The Bells Test, Figure Copying, Clock Drawing, Line Bisection, sobreposição de figuras, leitura e escrita e uma escala de avaliação comportamental de heminegligência (Catherine Bergego Scale - CBS). Essa bateria foi sensível para avaliar pacientes com AVC de hemisfério direito, sendo que $85 \%$ dos pacientes apresentaram heminegligência em pelo menos um teste. Os testes mais sensíveis foram o Bells Test e leitura. A VR Diagnostic test battery (VR-DiSTRO) é uma bateria de realidade virtual em que o computador simula o ambiente que foi desenvolvida para avaliar heminegligência com mais acurácia e rapidez. Essa bateria possui quatro testes: VR-Star Cancellation Test (VR-SCT), VR-Line Bisection (VR-LB), VR-Visual Extinction (VR-EXT), VRBaking Tray Task (VR-BTT). Também possui um módulo de reabilitação, como um jogo de computador (Fordell et al., 2011).

Nos artigos em que essas baterias foram utilizadas a única população estudada foi AVC. Mesmo nas baterias que envolvem outras tarefas na avaliação de heminegligência, os testes de cancelamento são os que mais identificam a síndrome em decorrência da busca/escaneamento que deve ser realizado pelo paciente. No que tange à análise comparativa entre estudos com pacientes pós-AVC e pós-TCE, de modo surpreendente, apenas uma parcela mínima dos estudos analisados, dois artigos, avaliava exclusivamente pacientes com o segundo quadro. Na medida em que a prevalência pode ser observada nos estudo de Mckenna, Cooke, Fleming, Jefferson e Ogden (2006), em que se verificou que a incidência de heminegligência em quadros de TCE é de 45,2\%, já Laurent-Vannier et al. (2006) identificaram 30\% de heminegligência em crianças com lesão de hemisfério direito, em que 32 de 41 crianças sofreram TCE, tal discrepância de quantidade de estudos por amostra era inesperada. Uma das possíveis explicações para este achado é que parece haver uma tendência de se examinar processos cognitivos específicos em pacientes pós-TCE a partir de testes de cancelamento, sem um interesse focado na possível identificação de uma determinada síndrome como a heminegligência. Por exemplo, na meta-análise de Mathias e Wheaton (2007), em que a autora pesquisou os instrumentos para avaliar processamento da atenção em amostras com TCE, e foram identificados 10 estudos 
que selecionaram tarefas de cancelamento com o intuito específico de avaliar acurácia, velocidade de processamento e busca visual. Além disso, tais estudos foram publicados entre 1992 e 2000, ou seja, há mais de uma década.

Por outro lado, em pesquisas com pacientes pós-AVC a investigação da atenção está frequentemente associada ao interesse em se diagnosticar heminegligência. Considerando-se que a frequência de heminegligência em AVC pode ser ao redor de $20 \%$ maior que no TCE, isto é, variando de $10 \%$ a 68,8\% (Azouvi et al., 2006; Bailey, Riddoch \& Crome, 2004; Halligan, Marshall \& Wade, 1990; Lee et al., 2004; Linden, Samuelsson, Skoog \& Blomstrand, 2005; Lopes, Ferreira, Carvalho, Cardoso \& André, 2007; Vanier et al., 1990), dessa forma não parece ser coerente a diferença de percentual de artigos encontrados significativamente maior de estudos envolvendo populações com doenças cerebrovasculares e somente dois estudos com TCE, já que em ambos os quadros a prevalência de heminegligência é alta. Dessa realidade sugere-se algumas hipóteses: talvez o TCE por ser uma lesão mais difusa e com lateralização menos evidente, a presença de heminegligência não fique tão exacerbada; os estudos podem estar mais preocupados com síndromes gerais como alterações perceptivas e/ou atencionais e menos com síndromes mais específica como heminegligência, mesmo que esta seja altamente prevalente em pacientes com TCE. Dessa forma, parece que avaliação da atenção concentrada visual em pacientes com TCE não está baseada no paradigma do cancelamento o que pode ocasionar uma reabilitação incompleta não levando em consideração essa possível disfunção.

Portanto, com base no levantamento dos artigos completos, observa-se que os testes de cancelamento com distratores são os mais sensíveis na identificação de heminegligência, tais como Bells Test e Star Cancellation. Podendo também ser usados na identificação de desatenção quando as omissões ocorrem no lado contralateral e ipsilesional à lesão, na identificação de estratégias, como identificar a coluna em que o paciente inicia, sendo que a busca que inicia pela direita é indício de heminegligência. E até como um teste para avaliar funções executivas, como organização e planejamento. Portanto, o uso de testes de cancelamento tanto lápis e papel quanto testes de cancelamento computadorizados, que parecem ser tão específicos e sensíveis e mais rápidos que os primeiros, demonstraram ser importantes para identificação de quadros de heminegligência e serem essenciais na avaliação neuropsicológica e para ter indícios de prognóstico.

\section{Considerações Finais}

Essa revisão sistemática da literatura possibilitou identificar 26 dos testes de cancelamento utilizados para avaliar o processamento atencional perceptivo em amostras de pacientes com AVC e TCE. Os instrumentos 
identificados mais utilizados nos artigos completos foram: Star Cancellation seguido do Line Bissection, Line Crossing e Letter Cancellation.

Foi possível verificar a escassez de artigos com TCE que enfoquem na avaliação da atenção com o uso de teste de cancelamento. Além de um pequeno número que abordam o uso do teste de cancelamento para avaliação de heminegligência. No entanto, para população com AVC pode-se observar uma ampla gama de pesquisas que enfocam tanto na avaliação, quanto nas estratégias utilizadas pelos pacientes e na reabilitação. Dessa forma, sugere-se o uso de teste de cancelamento para avaliação da atenção e heminegligência em ambos quadros neurológicos. Sabe-se das limitações desse estudo em especial quanto ao número de artigos não encontrados, pois buscou-se fazer um levantamento o mais abrangente possível, no entanto, foram encontrados artigos muito antigos que não estavam disponíveis online. De acordo com os resumos desses artigos que não foram lidos na integra não há informações diferentes daquelas apresentadas nos resultados do presente estudo. Por outro lado, a busca não foi delimitada por tempo para permitir que os estudos de referência pudessem ser encontrados. Desses, apenas três incluíam população com TCE e os outros 22 eram com AVC.

Para próximos estudos e intervenções ressalta-se a importância de pesquisas empíricas com instrumentos de cancelamento na avaliação de heminegligência pós-TCE, pois parece ser um quadro que está sendo pouco discutido e incluído nas avaliações neuropsicológicas da atenção e percepção. Por fim, mais estudos com utilização de paradigmas de cancelamento na avaliação de heminegligência com outras populações neurológicas e psiquiátricas devem ser conduzidas para identificação dos instrumentos utilizados nas avaliações cognitivas realizadas.

\section{Referências}

Agrell, B. M., Dehlin, O. I., \& Dahlgren C. J. (1997). Neglect in elderly stroke patients: a comparison of five tests. Psychiatry and Clinical Neurosciences, 51, 295-300.

Azouvi, P., Bartolomeo, P., Beis, J-M., Perennoud,D., Pradat-Diehl, P. \& Rousseaux, M. (2006). A battery of tests for the quantitative assessment of unilateral neglect. Restorative Neurology and Neuroscience, 24, 273-285.

Azouvi, P., Samuel, C., Louis-Dreyfus, A., Bernati, T., Bartolomeo, P., Beis, J. M., Chokron, S., Leclercq, M., Marchal, F., Martin, Y., De Montety, G., Olivier, S., Perennou, D., Pradat-Diehl, P., Prairial, C., Rode, G., Siéroff, E., Wiart, L., \& Rousseaux, M. (2002). Sensitivity of clinical and behavioural tests of spatial neglect after right hemisphere stroke. Journal of Neurology, Neurosurgery \& Psychiatry, 73, 160-166. 
Bailey, M. J., Riddoch, M. J., \& Crome, P. (2004) Test-retest stability of three tests for unilateral visual neglect in patients with stroke: Star Cancellation, Line Bisection, and the Baking Tray Task. Neuropsychological Rehabilitation, 14, 403-419.

Bailey, M. J., Riddoch, M. J., \& Crome, P. (2002). Treatment of visual neglect in elderly patients with stroke: a single-subject series using either a scanning and cueing strategy or a left-limb activation strategy. Journal of Physical Therapy, $82,782-797$.

Bailey, M. J., Riddoch, M. J., \& Crome, P. (2000). Evaluation of a test battery for hemineglect in elderly stroke patients for use by therapists in clinical practice. NeuroRehabilitation, 14, 139-150.

Beis, J. M., Keller, C., Morin, N., Bartolomeo, P., Bernati, T., Chokron, S., Leclercq, M., Louis-Dreyfus, A., Marchal, F., Martin, Y., Perennou, D., Pradat-Diehl, P., Prairial, C., Rode, G., Rousseaux, M., Samuel, C., Sieroff, E., Wiart, L., \& Azouvi, P. (2004). Right spatial neglect after left hemisphere stroke: qualitative and quantitative study. Neurology, 63, 1600-1605.

Broeren, J., Samuelsson, H., Stibrant-Sunnerhagen, K., Blomstrand, C., \& Rydmark, M. (2007). Neglect assessment as an application of virtual reality. Acta Neurologica Scandinavica, 116, 157-163.

Butler, B. C., Eskes, G. A., \& Vandorpe, R.A. (2004). Gradients of detection in neglect: comparison of peripersonal and extrapersonal space. Neuropsychologia, 42, 346-358.

Cassidy, T. P., Lewis, S., \& Gray, C. S. (1998). Recovery from visuospatial neglect in stroke patients. Journal of Neurology, Neurosurgery \& Psychiatry, 64, 555$-557$.

Chen-Sea, M. (2001). Unilateral neglect and functional significance among patients with stroke. Occupational Therapy Journal of Research, 21, 223-240.

Cumming, T. B., Plummer-D'Amato, P., Linden, T., \& Bernhardt, J. (2009). Hemispatial neglect and rehabilitation in acute stroke. Archives of Physical Medicine and Rehabilitation, 90, 1931-1936.

Draper, K., \& Ponsford, J. (2008). Cognitive Functioning Ten Years Following Traumatic Brain Injury and Rehabilitation. Neuropsychology, 22, 618-625.

Donovan, N. J., Kendall, D. L., Heaton, S. C., Kwon, S., Velozo, C. A., \& Duncan, P. W. (2008). Conceptualizing Functional Cognition in Stroke. Neurorehabilitation \& Neural Repair, 22, 122-135.

Echemendia, R. J., Putukian, M., Mackin, R. S., Julian, L., \& Shoss, N. (2001). Neuropsychological test performance prior to and following sports-related mild traumatic brain injury. Clinical Journal of Sport Medicine, 11, 23-31.

Fann, J. R., Hart, T., \& Schomer, K. G. (2009). Treatment for Depression after Traumatic Brain Injury: a systematic review. Journal of Neurotrauma, 26, 2383-2402.

Farner, L., Wagle, J., Engedal, K., Flekkøy, K. M., Wyller, T. B., \& Fure, B. (2010). Depressive symptoms in stroke patients: A 13 month follow-up study of patients referred to a rehabilitation unit. Journal of Affective Disorders, 127, 211-218 . 
Feijó, M. M., \& Portela, M. C. (2001). Variação no custo de internações hospitalares por lesões: os casos dos traumatismos cranianos e acidentes por armas de fogo. Caderno de Saúde Pública, Rio de Janeiro, 17, 627-637.

Ferber, S., \& Karnath, H-O. (2001). How to Assess Spatial Neglect: Line Bisection or Cancellation Tasks? Journal of Clinical and Experimental Neuropsychology, 23, 599-607.

Ferro J. (2001) Hyperacute cognitive stroke syndromes. Journal of Neurology, 248, 841-849.

Fordell, H., Bodin, K., Bucht, G., \& Malm, J. (2011). A virtual reality test battery for assessment and screening of spatial neglect. Acta Neurologica Scandinavica, 123, 167-174.

Fortis, P., Maravita, A., Gallucci, M., Ronchi, R., Grassi, E., Senna, I., Olgiati, E., Perucca, L., Banco, E., Posteraro, L., Tesio, L., \& Vallar, G. (2010). Rehabilitating patients with left spatial neglect by prism exposure during a visuomotor activity. Neuropsychology, 24, 681-697.

Friedman, P. J. (1992). The Star Cancellation Test in acute stroke. Clinical Rehabilitation, 6, 23-30.

Friedman, P. J. (1990). Spatial neglect in acute stroke: The line bisection test. Scandinavian Journal of Rehabilitation Medicine, 22, 101-106.

George, M. S., Mercer, J. S., Walker, R., \& Manly, T. (2008). A demonstration of endogenous modulation of unilateral spatial neglect: the impact of apparent time-pressure on spatial bias. Journal of the International Neuropsychological Society, 4, 33-41.

Gianotti, G., Messerli, P., \& Tissot, R. (1972). Qualitative analysis of unilateral spatial neglect in relation to laterality of cerebral lesions. Journal of Neurology, Neurosurgery, and Psychiatry, 35, 545-550.

Gottesman, R. F., Bahrainwala, Z., Wityk, R. L., \& Hillis, A. E. (2010). Neglect is more common and severe at extreme hemoglobin levels in right hemispheric stroke. Acta Neurologica Scandinavica, 21, 1641-1645.

Gottesman, R. F., Kleinman, J. T., Davis, C., Heidler-Gary, J., Newhart, M., Kannan, V., \& Hillis, A. E. (2008). Unilateral neglect is more severe and common in older patients with right hemispheric stroke. Neurology, 71, 1439-1444.

Halligan, P. W., \& Marshall, J. C. (1998). Visuospatial neglect: the ultimate deconstruction? Brain and Cognition, 37, 419-438.

Halligan, P. W., Burn, J. P., Marshall, J. C., \& Wade, D. T. (1992). Visuo-spatial neglect: Qualitative differences and laterality of cerebral lesion. Journal of Neurology, Neurosurgery \& Psychiatry, 55, 1060-1068.

Halligan, P. W., Marshall, J. C., \& Wade, D. T. (1990). Do visual field deficits exacerbate visuo-spatial neglect? Journal of Neurology, Neurosurgery \& Psychiatry, 53, 487-491.

Henry, J., \& Crawford, J. (2004). A meta-analytic review of verbal fluency performance in patients with traumatic brain injury. Neuropsychology, 18, 621-628.

Hills, E. C., \& Geldmacher, D. S. (1998). The effect of character and array type on visual spatial search quality following traumatic brain injury. Brain Injury, 12, 69-76. 
Hoffmann, M., Schmitt F., \& Bromley, E. (2009). Comprehensive cognitive neurological assessment in stroke. Acta Neurologica Scandinavica, 119, 162-171.

Jalas, M. J., Lindell, A. B., Brunila, T., Tenovuo, O., \& Hämäläinen, H. (2002). Initial rightward orienting bias in clinical tasks: Normal subjects and right hemispheric stroke patients with and without neglect. Journal of Clinical and Experimental Neuropsychology, 24, 479-490.

Jodzio, K., Lass, P., Nyka, W., Gasecki, D., Bandurski, T., \& Scheffler, J. (2002). Cerebral blood flow SPECT imaging in right hemisphere-damaged patients with hemispatial neglect. A pilot study. Nuclear medicine review. Central \& Eastern Europe: journal of Bulgarian, Czech, Macedonian, Polish, Romanian, Russian, Slovak, Yugoslav societies of nuclear medicine and Ukrainian Society of Radiology, 5, 49-51.

Keane, S., Turner, C., Sherrington, C., \& Beard, J. R. (2006). Use of fresnel prism glasses to treat stroke patients with hemispatial neglect. Archives of Physical Medicine and Rehabilitation, 87, 1668-1672.

Kim, D. Y., Ku, J., Chang, W. H., Park, T. H., Lim, J. Y., Han, K., Kim, I. Y., \& Kim, S. I. (2010). Assessment of post-stroke extrapersonal neglect using a three-dimensional immersive virtual street crossing program. Acta Neurologica Scandinavica, 121, 171-177.

Kim, J., Kim, K, Kim, D. Y., Chang, W. H., Park, C. I., Ohn, S. H., Han, K., Ku, J., Nam, S. W., Kim, I. Y., \& Kim, S. I. (2007). Virtual environment training system for rehabilitation of stroke patients with unilateral neglect: Crossing the virtual street. Cyberpsychology, behavior and social networking, 10, 7-15.

Kizony, R., \& Katz, N. (2002). Relationships between cognitive abilities and the process scale and skills of the assessment of motor process skills (AMPS) in patients with stroke. OTJR: Occupation, Participation and Health, 22, 82-92.

Ko, M. H., Han, S. H., Park, S. H., Seo, J. H., \& Kim, Y. H. (2008). Improvement of visual scanning after DC brain polarization of parietal cortex in stroke patients with spatial neglect. Neuroscience Letters, 448, 171-174.

Koch, G., Oliveri, M., Cheeran, B., Ruge, D., Lo, G. E., Salerno, S., Torriero, S., Marconi, B., Mori, F., Driver, J., Rothwell, J. C., \& Caltagirone, C. (2008). Hyperexcitability of parietal-motor functional connections in the intact left-hemisphere of patients with neglect. Brain, 131, 3147-3155.

Kollen, B., van de Port, I., Lindeman, E., Twisk, J., \& Kwakkel, G. (2005). Predicting improvement in gait after stroke: A longitudinal prospective study. Stroke, 36, 2676-2680.

Kwakkel, G., \& Kollen, B. (2007). Predicting improvement in the upper paretic limb after stroke: A longitudinal prospective study. Restorative neurology and neuroscience, $25,453-460$.

Kwakkel, G., Kollen, B., \& Twisk, J. (2006). Impact of time on improvement of outcome after stroke. Stroke. 37, 2348-2353.

Lafosse, C., Kerckhofs, E., Troch, M., \& Vandenbussche, E. (2003). Upper limb exteroceptive somatosensory and proprioceptive sensory afferent modulation of hemispatial neglect. Journal of Clinical and Experimental Neuropsychology, $25,308-323$. 
Larson, M.L., Demery, J. A., Cole, M., \& Perlstein, W. M. (2005). Cognitive control in closed head injury: Context maintence dysfunction or prepotent response inhibition deficit? Neuropsychology, 19, 578-590.

Laurent-Vannier, A., Chevignard, M., Pradat-Diehl, P., Abada, G., \& De Agostini, M. (2006). Assessment of unilateral spatial neglect in children using the Teddy Bear Cancellation Test. Developmental Medicine and Child Neurology, 48, 120-125.

Laurent-Vannier, A., Pradat-Diehl, P., Chevignard, M., Abada, G., \& De Agostini, M. (2003). Spatial and motor neglect in children. Neurology. 60, 202-207.

Lee, B. H., Kang, S. J., Park, J. M., Son, Y., Lee, K. H., Adair, J. C., Heilman, K. M., \& Na, D. L. (2004). The Character-line Bisection Task: A new test for hemispatial neglect. Neuropsychologia, 42, 1715-1724.

Leibovitch, F. S., Black, S. E., Caldwell, C. B., Ebert, P. L., Ehrlich, L. E., \& Szalai, J. P. (1998). Brain-behavior correlations in hemispatial neglect using CT and SPECT: The Sunnybrook Stroke Study. Neurology, 50, 901-908.

Lindell, A. B., Jalas, M. J., Tenovuo, O., Brunila, T., Voeten, M. J., \& Hämäläinen H. (2007). Clinical assessment of hemispatial neglect: Evaluation of different measures and dimensions. Archives of Clinical Neuropsychology, 21, 479-497.

Linden, T., Samuelsson, H., Skoog, I., \& Blomstrand, C. (2005). Visual neglect and cognitive impairment in elderly patients late after stroke. Acta Neurologica Scandinavica, 111, 163-168.

Lopes, M. A. L., Ferreira, H. P., Carvalho, J. C., Cardoso, L., \& André, C. (2007). Screening tests are not enough to detect hemineglect. Arquivos de Neuropsiquiatria, 65, 1192-1195.

Louie, S. W., Wong, S. K., \& Wong, C. M. (2009). Profiles of functional outcomes in stroke rehabilitation for Chinese population: A cluster analysis. NeuroRehabilitation, 25, 129-1235.

Lowery, N., Ragland, D., Gur, R. C., Gur, R. E., \& Moberg, P. J.(2004). Normative data for the symbol cancellation test in Young healthy adults. Applied Neuropsychology, 11, 216-219.

Maeshima, S., Truman, G., Smith, D. S, Dohi, N., Nakai, K., Itakura, T., \& Komai, N. (1997). Is unilateral spatial neglect a single phenomenon? A comparative study between exploratory-motor and visual-counting tests. Journal of Neurology, 244, 412-417.

Malhotra, P., Jäger, H. R., Parton, A., Greenwood, R., Playford, E. D., Brown, M. M., Driver, J., \& Husain, M. (2005). Spatial working memory capacity in unilateral neglect. Brain. 128, 424-435.

Manly, T., Woldt, K., Watson, P., \& Warburton, E. (2002). Is motor perseveration in unilateral neglect 'driven' by the presence of neglected left-sided stimuli? Neuropsychologia.40,1794-1803.

Mark, V. W., Woods, A. J., Ball, K. K., Roth, D. L., \& Mennemeier, M. (2004). Disorganized search on cancellation is not a consequence of neglect. Neurology, 63, 78-84.

Mark, V. W., \& Heilman, K. M. (1998). Diagonal spatial neglect. Journal of Neurology, Neurosurgery \& Psychiatry, 65, 348-352. 
Mark, V. W., \& Heilman K., M. (1997). Diagonal neglect on cancellation. Neuropsychologia, 35, 1425-1436.

Marshall, S. C., Grinnell, D., Heisel, B., Newall, A., \& Hunt, L. (1997). Attentional deficits in stroke patients: a visual dual task experiment. Archives of Physical Medicine and Rehabilitation, 78, 7-12.

Marshall, J. C., \& Halligan, P. W. (1996). Hemispheric antagonism in visuo-spatial neglect: A case study. Journal of the International Neuropsychological Society, 2, 412-418.

Mathias, J. L., \& Wheaton, P. (2007). Changes in attention and information-processing speed following severe traumatic brain injury: A meta-analytic review. Neuropsychology, 11, 212-223.

Mattingley, J. B., Berberovic, N., Corben, L., Slavin, M. J., Nicholls, M. E., \& Bradshaw, J. L. (2004). The greyscales task: a perceptual measure of attentional bias following unilateral hemispheric damage. Neuropsychologia, 42, 387-394.

McCarthy, M., Beaumont, J. G., Thompson, R., \& Pringle, H. (2002). The role of imagery in the rehabilitation of neglect in severely disabled brain-injured adults. Archives of Clinical Neuropsychology, 17, 407-422.

Mckenna, K., Cooke, D., Fleming, J., Jefferson, A., \& Ogden S. (2006). The incidence of visual perceptual impairment in patients with severe traumatic brain injury. Brain Injury, 20, 507-518.

Mesulam, M.-M. (1999). Spatial attention and neglect: Parietal, frontal and cingulated contributions to the mental representation and attentional targeting of salient extrapersonal events. Philosophical Transactions of the Royal Society London, 354, 1325-1346.

Mizuno, M. (1991). Neuropsychological characteristics of right hemisphere damage: investigation by attention tests, concept formation and change test, and self-evaluation task. Keio Journal of Medicine, 40, 221-234.

Na, D. L., Adair, J. C., Choi, S. H., Seo, D. W., Kang, Y., \& Heilman, K. M. (2000). Ipsilesional versus contralesional neglect depends on attentional demands. Cortex, 36, 455-467.

Na, D. L., Adair, J. C., Kang, Y., Chung, C. S., Lee, K. H., \& Heilman, K. M. (1999). Motor perseverative behavior on a line cancellation task. Neurology, 52, 1569-1576.

Na, D. L., Adair, J. C., Williamson, D. J. G., Schwartz, Ro. L., Haws, B., \& Heilman, K. M. (1998). Dissociation of sensory-attentional from motor-intentional neglect. Journal of Neurology, Neurosurgery \& Psychiatry, 64, 331-338.

National Institute of Health (1998). Rehabilitation of persons with traumatic brain injury. United States National Institutes of Health Consensus Statement, 16, 1-41 .

Niemeier, J. P. (2002). Visual imagery training for patients with visual perceptual deficits following right hemisphere cerebrovascular accidents: A case study presenting the Lighthouse Strategy. Rehabilitation Psychology, 47, 426-437.

Niemeier J. P. (1998). The Lighthouse Strategy: use of a visual imagery technique to treat visual inattention in stroke patients. Brain Injury, 12, 399-406. 
Nurmi, L., Kettunen, J., Laihosalo, M., Ruuskanen, E. I., Koivisto, A. M., \& Jehkonen, M. (2010). Right hemisphere infarct patients and healthy controls: evaluation of starting points in cancellation tasks. Journal of the International Neuropsychological Society, 16, 902-909.

Nys, G. M., de Haan, E. H., Kunneman, A., de Kort, P. L., \& Dijkerman, H. C. (2008). Acute neglect rehabilitation using repetitive prism adaptation: a randomized placebo-controlled trial. Restorative neurology and neuroscience, 26, 1-12.

Nys, G. M. S., Nijboer, T. C. W., \& de Haan, E. H. F. (2008). Incomplete ipsilesional hallucinations in a patient with neglect. Cortex: A Journal Devoted to the Study of the Nervous System and Behavior, 44, 350-352.

Nys, G. M. S., Seurinck, R., \& Dijkerman,H. D.(2008). Prism adaptation moves neglect-related perseveration to contralesional space. Cognitive and Behavioral Neurology, 21, 249-253.

Nys, G. M. S., Stuart, M., \& Dijkerman, C. H. (2010). Repetitive exploration towards locations that no longer carry a target in patients with neglect. Journal of Neuropsychology, 4, 33-45.

O'Neill, B., \& McMillan, T. M. (2004). The efficacy of contralesional limb activation in rehabilitation of unilateral hemiplegia and visual neglect: A baseline-intervention study. Neuropsychological Rehabilitation, 14, 437-447.

Piras, C., Forte, L. F., Peluso, C. M., Lima, E. M, \& Prandini, M. N. (2004). Estudo epidemiológico em unidade de terapia intensiva geral como resultado da adesão ao latin american brain injury consortium. Revista Brasileira Terapia Intensiva, 16, 164-169.

Polanowska, K., Seniów, J., Paprot, E., Leśniak, M., \& Członkowska, A. (2009). Left-hand somatosensory stimulation combined with visual scanning training in rehabilitation for post-stroke hemineglect: A randomised, double-blind study. Neuropsychological Rehabilitation, 19, 364-382.

Potter, J., Deighton, T., Patel, M., Fairhurst, M., Guest, R., \& Donnelly, N. (2000). Computer recording of standard tests of visual neglect in stroke patients. Clinical Rehabilitation, 14, 441-446.

Punt, T. D., Kitadono, K., Hulleman, J., Humphreys, G. W., \& Riddoch, M. J. (2008). From both sides now: crossover effects influence navigation in patients with unilateral neglect. Journal of Neurology, Neurosurgery \& Psychiatry, 79, 464-466.

Rorsman, I., Magnusson, M., \& Johansson, B. B. (1999). Reduction of visuo-spatial neglect with vestibular galvanic stimulation. Scandinavian Journal of Rehabilitation Medicine, 31, 117-124.

Rovira, A., Grive, E., Rovira, A., \& Alvarez-Sabin, J. (2005). Distribution territories and causative mechanisms of ischemic stroke. European Radiology, 15, 416$-426$.

Rusconi, M. L., Maravita, A., Bottini, G., \& Vallar, G. (2002). Is the intact side really intact? Perseverative responses in patients with unilateral neglect: A productive manifestation. Neuropsychologia, 40, 594-604.

Sarri, M., Greenwood, R., Kalra, L., Papps, B., Husain, M., \& Driver, J. (2008). Prism adaptation aftereffects in stroke patients with spatial neglect: pathologi- 
cal effects on subjective straight ahead but not visual open-loop pointing. Neuropsychologia, 46, 1069-1080.

Schmitter-Edgecombe, M., \& Kibby, M. K. (1998). Visual selective attention after severe closed head injury. Journal of the international neuropsychological society, 4, 144-159.

Schubert, F., \& Spatt, J. (2001). Double dissociations between neglect tests: possible relation to lesion site. European Journal of Neurology, 45, 160-164.

Siéroff, E., Decaix, C., Chokron, S., \& Bartolomeo, P. (2007). Impaired Orienting of Attention in Left Unilateral Neglect: A Componential Analysis. Neuropsychology, 21, 94-113.

Song, W., Du, B., Xu, Q., Hu, J., Wang, M., \& Luo, Y. (2009). Low-frequency transcranial magnetic stimulation for visual spatial neglect: a pilot study. Journal of Rehabilitation Medicine, 41, 162-165.

Spalletta, G., Serra, L., Fadda, L., Ripa, A., Bria, P., \& Caltagirone, C. (2007). Unawareness of motor impairment and emotions in right hemispheric stroke: A preliminary investigation. International Journal of Geriatric Psychiatry, 22, 1241-1246.

Speedie, L. J., Wertman, E., Verfaellie, M., Butter, C., Silberman, N., Liechtenstein, M., \& Heilman, K. M. (2002). Reading direction and spatial neglect. Cortex. $38,59-67$.

Suzuki, M., Omori, M., Hatakeyama, M., Yamada, S., Matsushita, K., \& Iijima, S. (2006). Predicting recovery of upper-body dressing ability after stroke. Archives of Physical Medicine and Rehabilitation, 87, 1496-1502.

Strauss, E., Sherman, E., \& Spren, O. (2006). A compendium of Neuropsychological Tests: Administration, Norms and Commentary (3a ed.). New York, USA: Oxford.

Tanaka, T., Ifukube, T., Sugihara, S., \& Izumi, T. (2010). A case study of new assessment and training of unilateral spatial neglect in stroke patients: Effect of visual image transformation and visual stimulation by using a Head Mounted Display system (HMD). Journal of NeuroEngineering and Rehabilitation, 7, 1-8 .

Tanaka, T., Sugihara, S., Nara, H., Ino, S., \& Ifukube, T. (2005). A preliminary study of clinical assessment of left unilateral spatial neglect using a head mounted display system (HMD) in rehabilitation engineering technology. Journal of NeuroEngineering and Rehabilitation, 2, 1-9.

Tatemichi, T. K., Desmond, D. W., Stem,Y., Paik, M., Sano, M., \& Bagiella, E. (1994). Cognitive impairment after stroke: frequency, patterns, and relationship to functional abilities. Journal of Neurology, Neurosurgery, and Psychiatry, 57, 202-207.

van Kessel, M. E., van Nes, I. J., Brouwer, W. H., Geurts, A. C., \& Fasotti, L. (2010). Visuospatial asymmetry and non-spatial attention in subacute stroke patients with and without neglect. Cortex. 46, 602-612.

van Nes, I. J., van Kessel, M. E., Schils, F., Fasotti, L., Geurts, A. C., \& Kwakkel, G. (2009). Is visuospatial hemineglect longitudinally associated with postural imbalance in the postacute phase of stroke? Neurorehabilitation \& Neural Repair, 23, 819-824. 
Vanier, M., Gauthier, L., Lambert, J., Pepin, E. P., Robillard, A., Dubouloz, C. J., Gagnon, R., \& Joannette, Y. (1990). Evaluation of left visuospatial neglect: Norms and discrimination power of two tests. Neuropsychology, 4, 87-96.

Vossel, S., Eschenbeck, P., Weiss, P. H., Weidner, R., Saliger, J., Karbe, H., \& Fink, G. R. (2011). Visual extinction in relation to visuospatial neglect after right-hemispheric stroke: quantitative assessment and statistical lesion-symptom mapping. Journal of Neurology, Neurosurgery \& Psychiatry, 82, 862-868.

Vuilleumier, P. O., \& Rafal, R. D. (2000). A systematic study of visual extinction. Between-and within-field deficits of attention in hemispatial neglect. Brain, $123,1263-1279$.

Wade, D. T., Wood, V. A., \& Hewer, R. L. (1988). Recovery of cognitive function soon after stroke: A study of visual neglect, attention span and verbal recall. Journal of Neurology, Neurosurgery \& Psychiatry, 51, 10-13.

Woods, A. J., \& Mark, V. W. (2007). Convergent validity of executive organization measures on cancellation. Journal of Clinical and Experimental Neuropsychology, 29, 719-723.

Yin, Y., Li, X., Li, Y., Gu, H., Han, C., \& Liu, H. (2009). Preliminary clinical study in patients with hemispatial neglect after stroke by neglect test battery and 99mTc-ECD single-photon emission computed tomography. Nuclear Medicine and Biology, 36, 467-475. 